\title{
IPrep - Automated Serial-Section Broad-Ion-Beam Tomography
}

\author{
T. Hosman, S. Coyle, A. Abbott, M. Olvera, M. Hassel-Shearer and J.A. Hunt
}

Gatan Inc., 5794 W. Las Positas Blvd., Pleasanton, CA 94588 USA

Tomographic reconstruction of a wide range of materials and biological samples can be accomplished via serial-section broad-ion-beam tomography (SSBIB) - alternately ion-polishing away material and imaging the remaining polished surface with optical or scanning electron microscopy (SEM) [1]. Under computer control it is practical to acquire 2-D images of hundreds of sections without user intervention and assemble them for 3-D visualization and measurements [2,3]. In general, the resulting 3-D images permit significantly better understanding of the structure of complex systems than would be possible with a few strategically acquired 2-D images acquired at various depths. Herein we report on the IPrep II tool and what we believe is the first general implementation of the SSBIB technique.

Broad-ion-beam milling can efficiently acquire tomographic data of several $\mathrm{mm}^{\wedge} 2$ areas because of its intense and large-diameter beam size. The low $\mathrm{keV}$ ions typically employed with BIB milling results in low surface damage and permits excellent imaging and analytical analysis (e.g., EDS/EBSD/CL) as compared to FIB methods [3]. The IPrep II milling system is based on the Gatan PECS II system and is capable of milling with noble gases from $8 \mathrm{keV}$ down to $100 \mathrm{eV}$. Milling rates in early experiments with $8 \mathrm{keV} \mathrm{Ar}$ ions have been measured at $>1 \mathrm{e} 6 \mathrm{um}^{\wedge} 3 /$ hour on tungsten carbide and $>3.5 \mathrm{e} 6 \mathrm{um}^{\wedge} 3 /$ hour with silicon devices. Final polishing with $1 \mathrm{keV}$ Ar ions reduces milling rates on silicon devices to $\sim 1 \mathrm{e} 5$ $\mathrm{um}^{\wedge} 3 /$ hour but further reduces milling artifacts. Milling rates herein are only quoted for the imaged volume; milled volume is many times larger.

Automation of the serial-sectioning process is required to make SSBIB practical. Specific challenges addressed with IPrep II include: (1) high-speed material removal while maintaining high surface quality and minimizing milling artifacts; (2) preventing contamination of the SEM from $\mathrm{mm}^{\wedge} 3$ of sputtered material over the course of a tomogram; (3) control over the repeatability and uniformity of increments in $\mathrm{Z}$ (polishing depth); (4) maintaining precise alignment of the sample in XYZ, rotation and both tilt axes while the sample is safely and reliably translated between polishing and imaging; and (5) accommodating planar analysis and analysis techniques requiring high-sample-tilt such as EBSD.

In the IPrep II implementation, the BIB system is mounted to the side of an SEM chamber (rather than inside) and isolated by a gate valve to prevent contamination of the SEM system from milling effluent. The sample is exchanged between the chambers by a linear transfer stage. A dock placed on the SEM stage receives the sample and clamps it kinematically for highly-repeatable alignment between successive sample exchanges. Two receiving docks have been developed - a planar dock for standard delayering (e.g., imaging, EDS \& CL spectrum imaging) and a $70^{\circ}$-inclined dock for EBSD analysis.

The schematic in Figure 1 shows the sample in the IPrep II chamber in the raised position and being milled. A reflection light microscope can be used to image the surface before and after milling. During milling the sample is rotated to reduce shadowing artifacts and the temperature can be cooled/regulated to prevent heating damage. After milling, the sample is lowered and a gripper on the transfer stage moves the sample into the SEM chamber as shown in Figure 2. A receiving dock mounted to the SEM stage is moved in position and the sample is exchanged with essentially no force on the SEM stage. SEM analysis is then performed and the process is repeated until the desired analysis depth is reached. 
References:

[1] Winiarski et al., (2016). Broad Ion Beam Serial Section Tomography. Manuscript in preparation.

[2] Burnett et al. (2016). Large volume serial section tomography by Xe Plasma FIB dual beam microscopy. Ultramicroscopy 161, 119-129.

[3] Gholinia et al., (2016). Automated three dimensional broad ion beam milling acquisition and analysis. To be presented at Royal Microscopy Society-EBSD, Manchester, U.K.

[4] The authors thank Philip J. Withers, Ali Gholinia and their colleagues at The University of Manchester School of Materials for their collaboration prior to and during the development of IPrep II.



Figure 1. IPrep II attached to an SEM. Sample in BIB milling position and IPrep vacuum isolated.

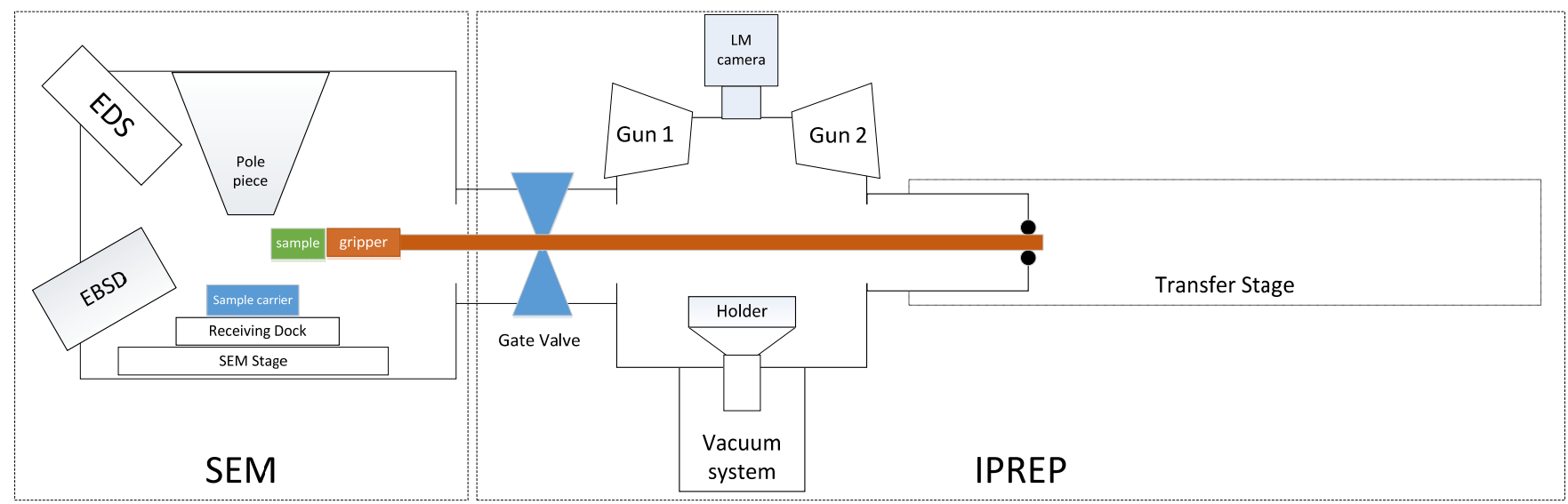

Figure 2. Sample is being transferred from the BIB milling chamber to the SEM chamber.
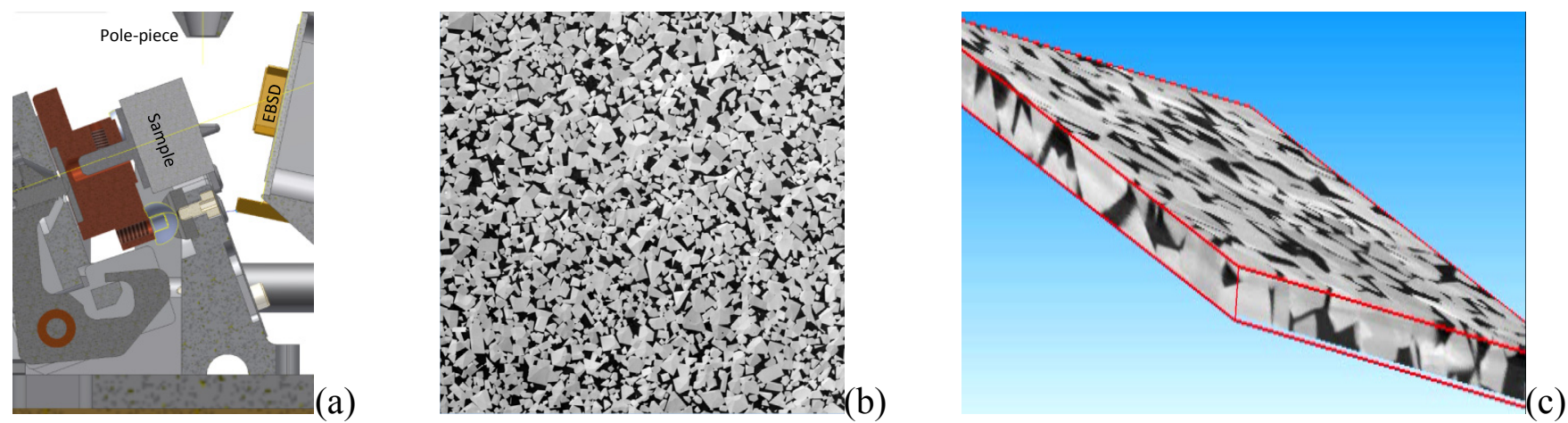

Figure 3. (a) Cross-section of dock \& sample in EBSD position. (b) Image of IPrep II BIB-milled WC sample $(0.4 \mathrm{~mm} \times 0.4 \mathrm{~mm}$ shown). (c) Sub-volume $(6 \%)$ of reconstructed tomogram. 